\title{
DOA ESTIMATION OF NONPARAMETRIC SPREADING SPATIAL SPECTRUM BASED ON BAYESIAN COMPRESSIVE SENSING EXPLOITING INTRA-TASK DEPENDENCY
}

\author{
Si Qin, Qisong Wu, Yimin D. Zhang and Moeness G. Amin
}

\author{
Center for Advanced Communications, Villanova University, Villanova, PA 19085, USA
}

\begin{abstract}
For spatially distributed targets encountered in radar and sonar applications, direct application of subspace-based methods usually do not lead to an accurate estimation of the direction and angular extent of the signal arrivals. If the spatial distribution of the targets can be parameterized with a known model $a$ priori, the direction-of-arrival (DOA) estimation problems can be simplified as parameter estimation problems. However, these methods do not apply when the targets are not parameterizable. Motivated by this fact, we propose an effective approach for the DOA estimation of nonparametric spatially extended targets. In the proposed approach, the spatially extended targets are modeled as a continuous sparse structure, which are effectively estimated using the Bayesian compressive sensing techniques based on a paired spike-and-slab prior accounting for the angular target spread. In particular, the problem is examined under a collocated multiple-input multiple-output (MIMO) radar platform. Signal transmission at multiple coprime transmit frequencies are also considered to achieve increased degreesof-freedom. The group sparsity of the targets across different frequencies is exploited to achieve improved DOA estimation performance.
\end{abstract}

Index Terms - Multiple-input multiple-output (MIMO) radar, Bayesian compressive sensing, direction-of-arrival (DOA) estimation, coprime frequency, sum coarray

\section{INTRODUCTION}

Direction-of-arrival (DOA) estimation is an important technique in radar and sonar applications to determine the spatial spectrum of impinging electromagnetic and acoustics waves. Subspace-based approaches, such as MUSIC and ESPRIT [1, 2], are considered effective to achieve high-resolution DOA estimations for uncorrelated point sources. When the signals are coherent, the covariance matrix becomes rank-deficient and, thereby, necessitates preprocessing using, e.g., spatial smoothing [3], to restore the rank of the covariance matrix before these subspace-based techniques are applied.

In this paper, we consider the problem of DOA estimation of multiple spatially extended targets in a collocated multipleinput multiple-output (MIMO) radar platform, which allows effective construction of a sum coarray aperture from the transmit and receive arrays [4]. The MIMO array structure also permits exploiting multiple frequencies, thus achieving

This work was supported in part by the Office of Naval Research (ONR) under grant N00014-13-1-0061. further enhanced degrees-of-freedom (DOFs). Spatially extended targets are commonly encountered in radar and sonar applications depending on the target size, measurement range, and propagation environments [5].

Several methods exist for DOA estimation of signals (targets) that exhibit angular spread. In [6], a MUSIC-type distributed signal parameter estimator (DSPE) is developed to estimate spatially distributed sources with a parametric angular cross-correlation kernel. The maximum likelihood technique is used in [7] to estimate the parameters of the angular distribution. Another algorithm, termed dispersed signal parametric estimation (DISPARE), is proposed in [8] under different angular distributions. Recently, a MUSIC-based method is introduced in [9] that uses coprime arrays to estimate spatially distributed sources for certain parametric models introduced in [10]. All these approaches assume a priori knowledge of the distribution of the angular spread so that the DOA estimation problem can be cast as a simpler parameter estimation problem. The assumptions of parametric distribution and its a priori knowledge, however, are valid only in some specific circumstance. There are several methods for DOA estimation of nonparametric spreading sources [11, 12], but they are only applicable when the distributed signal components are incoherent.

In this paper, compressive sensing (CS) methods are used for DOA estimation of angular spreading targets. The proposed technique applies to signals with both coherent and incoherent signal components. CS techniques [13] have the capability of recovering signals from a small number of measurement samples with a high probability, provided that the signals can be sparsely represented in some known domain as typical the case in DOA estimations. When multiple observations correspond to the same target locations but with different scattering coefficients, e.g., by using multiple sensing frequencies [14, 15], different polarizations [16], multi-static observations $[17,18]$, or multipath propagation $[19,20]$, such problem can further be cast into a group sparse reconstruction problem for improved performance.

Among the many CS algorithms that are capable to recover sparse and group sparse signals, we use the recently developed multi-task Bayesian compressive sensing techniques that accounts for inter- and intra-task dependencies [21]. This method falls into the general class of multi-task Bayesian compressive sensing [22-24] which generally yield enhanced capability and improved performance as compared to the other group sparse reconstruction methods. In addition, sparse Bayesian learning algorithms have the flexibility to effectively account for different signal and observation structures through the exploitation of modified priors. As a result, Bayesian learning based compressive sensing techniques 
have been successfully applied with a desirable performance in diverse areas of DOA estimation [25], synthetic aperture radar (SAR) and inverse SAR (ISAR) imaging [17, 21], timefrequency analysis [26, 27], and radar clutter suppression $[28,29]$.

To consider the underlying DOA estimation through the inter- and intra-task dependencies, scattering signals belong to the same group of spread coefficients are considered as a contiguous cluster with intra-task correlation in the spatial domain, whereas the fact that different sensing frequencies or polarizations share to the same target spatial support is cast as inter-task correlation between these observations. Note that the angular spread does not have a known size and structure in a priori and, therefore, cannot be treated in a typical group sparse context.

The problem of DOA estimation is considered in this paper in a collocated nested MIMO platform, which achieves a large virtual aperture and high number of degrees-of-freedom (DOF) under sum coarray equivalence [30-32]. It is shown in $[33,34]$ that the nested coarray can be cast as a special case of the generalized coprime array structure, and the result derived in this paper can be applied to the generalized coprime array as well. We further examine the case when narrowband waveforms are emitted in multiple coprime frequencies, thus achieving an increased number of DOFs, offering a greater flexibility in system design and the capability of configuration adaptivity to the application environment.

Notations: We use lower-case (upper-case) bold characters to denote vectors (matrices). $p(\cdot)$ denotes the probability density function (pdf) and $p(x \mid-)$ denotes the conditional pdf of random variable $x$, given other parameters. $\mathcal{C N}(x \mid a, b)$ denotes that random variable $x$ follows a complex Gaussian distribution with mean $a$ and variance $b$. In addition, $(\cdot)^{T}$ denotes transpose, and $\mathbf{I}_{N}$ denotes the $N \times N$ identity matrix. Furthermore, o denotes element-wise (Hadamard) multiplication, $\otimes$ implies the Kronecker product, and $\delta(x)$ is the Dirac delta function of $x$.

\section{SIGNAL MODEL}

\subsection{Nonparametric spatially extended targets}

Consider spatially extended targets as the collection of spatially contiguous point ones. In particular, target $q$ spans an angular section $\theta \in \boldsymbol{\theta}_{q}=\left\{\theta_{q 1}, \ldots, \theta_{q P_{q}}\right\}$, where $P_{q}$ denotes the number of discretized grids that are contiguous over the angular span $\boldsymbol{\theta}_{q}$. The corresponding scattering coefficients at time instant $t$ are respectively denoted as $\rho_{q 1}(t), \ldots, \rho_{q P_{q}}(t), t=1, \ldots, T$, where $T$ is the number of temporal samples. The coefficients are assumed to take arbitrary values and thus are not parameterizable.

\subsection{MIMO system with single frequency band signals}

We first consider the single frequency band case. Let a nested MIMO system consist of an $M$-element transmit uniform linear array (ULA) with an interelement spacing $d$ and an $N$-element receive ULA with interelement spacing $M d$, as shown in Fig. 1. $d$ is usually chosen to be $\lambda / 2$, where $\lambda$ is the wavelength. The transmit and receive arrays are collocated, i.e., targets are observed at the same direction by both transmit and receive arrays.

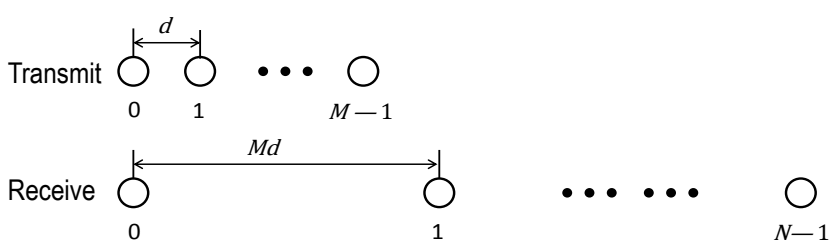

Fig. 1. A nested MIMO system.

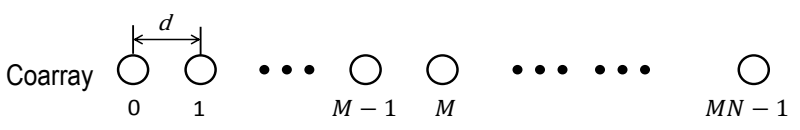

Fig. 2. The sum coarray of the MIMO system.

Assume that the $M$ transmit antennas emit $M$ temporally orthogonal narrowband waveforms. For a scene with $Q$ spatially spreading targets, the matched filter at the output of the $n$th receive antenna, corresponding to the $m$ th transmit waveform, is expressed as

$$
\tilde{x}_{m, n}(t)=\sum_{q=1}^{Q} \sum_{p=1}^{P_{q}} a_{t_{(m)}}\left(\theta_{q p}\right) a_{r_{(n)}}\left(\theta_{q p}\right) \rho_{q p}(t)+n_{m, n}(t)
$$

where

$$
\begin{aligned}
& a_{t_{(m)}}\left(\theta_{q p}\right)=e^{\frac{-j 2 \pi m d}{\lambda} \sin \left(\theta_{q p}\right),} \\
& a_{r_{(n)}}\left(\theta_{q p}\right)=e^{\frac{-j 2 \pi n M d}{\lambda} \sin \left(\theta_{q p}\right),}
\end{aligned}
$$

and $n_{m, n}(t)$ is the additive noise which is assumed to be independent and identically distributed (i.i.d.) random variable following the complex Gaussian distribution $\mathcal{N} C\left(0, \sigma_{n}^{2}\right)$. Stacking them for all values of $m$ and $n$ into a vector yields

$$
\begin{aligned}
\tilde{\mathbf{x}}(t)= & {\left[\tilde{x}_{1,1}(t), \tilde{x}_{2,1}(t), \ldots, \tilde{x}_{M, 1}(t), \tilde{x}_{1,2}(t), \tilde{x}_{2,2}(t), \ldots,\right.} \\
& \left.\tilde{x}_{M, 2}(t), \ldots, \tilde{x}_{1, N}(t), \tilde{x}_{2, N}(t), \ldots, \tilde{x}_{M, N}(t)\right]^{T} \\
= & \sum_{q=1}^{Q} \sum_{p=1}^{P_{q}} \mathbf{a}_{r}\left(\theta_{q p}\right) \otimes \mathbf{a}_{t}\left(\theta_{q p}\right) \rho_{q p}(t)+\mathbf{n}(t) \\
= & \sum_{q=1}^{Q} \sum_{p=1}^{P_{q}} \mathbf{a}\left(\theta_{q p}\right) \rho_{q p}(t)+\mathbf{n}(t)
\end{aligned}
$$

where

$$
\begin{aligned}
& \mathbf{a}_{t}\left(\theta_{q p}\right)=\left[a_{t_{(1)}}\left(\theta_{q p}\right), a_{t_{(2)}}\left(\theta_{q p}\right), \ldots, a_{t_{(M)}}\left(\theta_{q p}\right)\right]^{T}, \\
& \mathbf{a}_{r}\left(\theta_{q p}\right)=\left[a_{r_{(1)}}\left(\theta_{q p}\right), a_{r_{(2)}}\left(\theta_{q p}\right), \ldots, a_{r_{(N)}}\left(\theta_{q p}\right)\right]^{T}
\end{aligned}
$$

are the transmit and receive steering vectors corresponding to $\theta_{q p}$, respectively, $\mathbf{a}\left(\theta_{q p}\right)=\mathbf{a}_{r}\left(\theta_{q p}\right) \otimes \mathbf{a}_{t}\left(\theta_{q p}\right)$ is the equivalent steering vector of the sum coarray, and $\mathbf{n}(t)=$ $\left[n_{1,1}(t), n_{2,1}(t), \ldots, n_{M, N}(t)\right]^{T}$ is the noise vector. As a result of Eqn. (2), the output of the receiver matched filters, after combining redundant sensor lags, yields an $M N$-element sum coarray which is uniform linear, as illustrated in Fig. 2.

\subsection{MIMO system with multiple coprime frequencies}

We further extend the single frequency band signals to a multiple frequency band case, where the frequencies are chosen to 
be mutually coprime. ULAs with coprime frequencies have been shown to provide significant increase of the available DOFs for enhanced DOA estimation capability [14, 15].

For narrowband waveforms constructed with a center frequency $f_{l}$, where $l=1, \ldots, L$, with $L$ denoting the number of frequency bands, the received signal of the sum coarray can be similarly expressed as

$$
\mathbf{x}^{(l)}(t)=\sum_{q=1}^{Q} \sum_{p=1}^{P_{q}} \mathbf{a}^{(l)}\left(\theta_{q p}\right) \rho_{q p}^{(l)}(t)+\mathbf{n}^{(l)}(t) .
$$

Note that $\rho_{q p}^{(l)}(t)$ does not vary with the receive antennas but is generally frequency-dependent due to the different target scattering characteristics and propagation phase delays [14]. In addition, $\mathbf{a}^{(l)}\left(\theta_{q p}\right)=\mathbf{a}_{r}^{(l)}\left(\theta_{q p}\right) \otimes \mathbf{a}_{t}^{(l)}\left(\theta_{q p}\right)$ is the steering vector of the sum coarray corresponding to spatial angle $\theta_{q p}$ and frequency $f_{l}$, expressed as

$\mathbf{a}^{(l)}\left(\theta_{q p}\right)=\left[1, e^{-j \frac{2 \pi D}{\lambda_{l}} \sin \left(\theta_{q p}\right)}, \ldots, e^{-j \frac{2 \pi(M N-1) D}{\lambda_{l}} \sin \left(\theta_{q p}\right)}\right]^{T}$

where $\lambda_{l}=c / f_{l}$ denotes the wavelength corresponding to $f_{l}$, and $c$ is the velocity of wave propagation. Note that the interelement spacing of the sum coarray is denoted as $D$. The additive noise vectors $\mathbf{n}^{(l)}(t), l=1, \ldots, L$, are assumed to be uncorrelated to each other with their respective variances $\left[\sigma_{n}^{(l)}\right]^{2}$. The use of coprime frequencies minimizes the overlaps between the yielded virtual sensor positions in $\mathbf{a}^{(1)}, \ldots, \mathbf{a}^{(L)}$. Let the interelement spacing $D$ of the sum coarray be an integer multiple of the half-wavelength at all the coprime frequencies, i.e., $L_{l}=2 D / \lambda_{l}, l=1, \ldots, L$, are mutually coprime integers. As such, the ULA is spatially undersampled at each frequency. In this case, we can rewrite the steering vectors in a frequency-independent form for better clarity,

$$
\mathbf{a}^{(l)}\left(\theta_{q p}\right)=\left[1, e^{-j L_{l} \pi \sin \left(\theta_{q p}\right)}, \ldots, e^{-j L_{l}(M N-1) \pi \sin \left(\theta_{q p}\right)}\right]^{T} .
$$

\section{BAYESIAN COMPRESSIVE SENSING WITH INTER- AND INTRA-TASK DEPENDENCIES}

Denote $P=\Sigma_{q=1}^{Q} P_{q}$ as the total number of nonzero entries in the descritized spatial angles, the received signals can be expressed as the following multiple measurement vector (MMV) model,

$$
\mathbf{x}^{(l)}(t)=\mathbf{A}^{(l)} \mathbf{s}^{(l)}(t)+\mathbf{n}^{(l)}(t), l \in[1, \ldots, L],
$$

where $\mathbf{A}^{(l)}=\left[\mathbf{a}^{(l)}\left(\theta_{11}\right), \ldots, \mathbf{a}^{(l)}\left(\theta_{Q P_{Q}}\right)\right]$ is an $M N \times P$ matrix and $\mathbf{s}^{(l)}(t)=\left[\rho_{11}^{(l)}(t), \ldots, \rho_{Q P_{Q}}^{(l)}(t)\right]^{T}$ is a $P \times 1$ vector. In addition, the DOAs of the signals can be sparsely represented in the spatial domain over the entire angular grids as

$$
\mathbf{x}^{(l)}(t)=\mathbf{B}^{(l)} \mathbf{r}^{(l)}(t)+\mathbf{n}^{(l)}(t), l \in[1, \ldots, L],
$$

where $\mathbf{B}^{(l)}$ is defined as the collection of steering vectors over the entire possible girds $\theta_{\mathbf{g}}^{1}, \ldots, \theta_{\mathbf{g}}^{G}$, for $G \gg P$. Then, the angle positions of the signal arrivals are indicated by the nonzeros entries in $\mathbf{r}^{(l)}$, whose values describe the target scattering coefficients. Note that, $\mathbf{r}^{(l)}$ exhibits a group sparsity across the $L$ frequencies, because they share the same sparse pattern or the nonzero entry positions, but the scattering coefficients generally differ. Therefore, we can reconstruct the signals using group CS techniques. As we discussed earlier, in this paper, we use the MT-BCS algorithm exploiting inter- and intra-task dependencies [21], in view of continuity of spreading spatial spectrum, based on a paired spike-andslab prior [35].

Consider this problem as a clustered sparse reconstruction problem with $L$ tasks, each consisting of $G$ grids. To encourage the group sparsity described in (7), we place a spike-andslab prior to $\mathbf{r}^{(l)}[21,35]$, i.e.,

$$
p\left(\mathbf{r}^{(l)} \mid \boldsymbol{\pi}, \boldsymbol{\beta}\right)=\prod_{i=1}^{G}\left[\left(1-\pi_{i}\right) \delta\left(r_{i l}\right)+\pi_{i} \mathcal{C N}\left(r_{i l} \mid 0, \beta_{i}^{-1}\right)\right]
$$

where $r_{i l}$ is the $i$ th element of $\mathbf{r}^{(l)}$ and $\pi_{i}$ determines the prior probability of nonzero elements. That is, a large weight $\pi_{i}$ corresponds to a high probability for the $r_{i l}$ to take a nonzero value, whereas a small $\pi_{i}$ tends to generate a zero entry. In addition, $\beta_{i}$ is the precision (reciprocal of the variance) of the Gaussian distribution.

To make the Bayesian inference analytical in (8), which involves the delta function, we assume a Gaussian random vector $\boldsymbol{\eta}^{(l)}=\left[\eta_{1 l}, \ldots, \eta_{G l}\right]^{T}$ with $l=1, \ldots, L$ and a Bernoulli random vector $\mathbf{z}=\left[z_{1}, \ldots, z_{G}\right]^{T}$. Then, the $\mathbf{r}^{(l)}$ can be expressed as the element-wise product of the latent vectors $\boldsymbol{\eta}^{(l)}$ and $\mathbf{z}$ forms a new random vector that follows the pdf in (8), i.e.,

$$
\mathbf{r}^{(l)}=\boldsymbol{\eta}^{(l)} \circ \mathbf{z}
$$

where the following paired spike-and-slab prior is introduced,

$$
p\left(\boldsymbol{\eta}^{(l)}, \mathbf{z}\right)=\prod_{i=1}^{G}\left[\mathcal{C N}\left(\eta_{i l} \mid 0, \beta_{i}^{-1}\right)\right]^{z_{i}} \pi_{i}^{z_{i}}\left(1-\pi_{i}\right)^{1-z_{i}} .
$$

It is noted that the group sparsity is characterized by the same $\mathbf{z}$ across the $L$ frequencies, i.e., signals at all frequencies correspond to the same DOA positions. On the other hand, the scattering coefficients, denoted as $\boldsymbol{\eta}^{(l)}$, generally take different values for each frequency.

Without loss of generality, a Gaussian prior is placed on the additive noise in (7) as

$$
\mathbf{n}^{(l)}(t) \sim \mathcal{C N}\left(\mathbf{n}^{(l)}(t) \mid \mathbf{0}, \alpha_{l}^{-1} \mathbf{I}_{M N}\right)
$$

To acquire the trackable and analytical posterior distribution of $\beta_{i}$ and $\alpha_{l}$, we place respective Gamma priors, which are conjugate to the Gaussian distribution, on both of them.

Considering the continuity of spreading spatial spectrum, we place a cluster prior based on the intra-task relationship to improve the estimate performance. Similar to the approaches described in $[21,35]$, as illustrated in Fig. 3, we categorize the relationship into three different cluster patterns, and denote $\kappa_{i}$ as the number of nonzero neighboring blocks for the $i$ th 


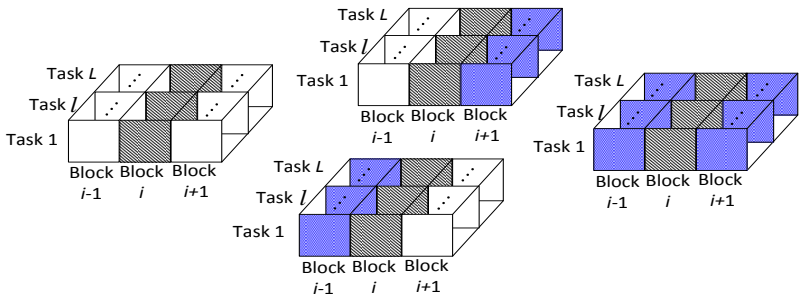

(a) Pattern 0

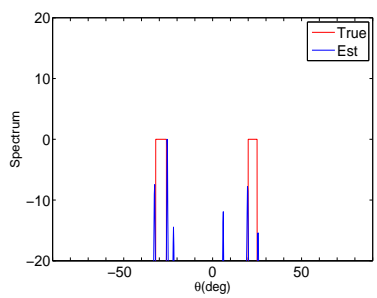

(a) MUSIC

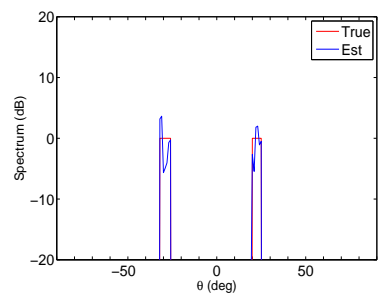

(b) Proposed approach

Fig. 3. Three cluster patterns.

block. In Pattern 0 ("strong rejection"), both neighboring blocks are zero valued, i.e., $\kappa_{i}=0$, and the $i$ th block would take a zero value with a high probability, since the targets of interest are assumed to be spatially spread. In this case, $e_{0}<$ $g_{0}$ is assumed in the prior $\operatorname{Beta}\left(e_{0}, g_{0}\right)$ to encourage a small value of $\pi_{i}^{(0)}$ so as to reject this block. In Pattern 1 ("weak rejection"), one of the neighboring blocks is zero valued, i.e., $\kappa_{i}=1$. In this case, the probability for the $i$ th block to be zero is fair, and $e_{1}=g_{1}$ is used in $\operatorname{Beta}\left(e_{1}, g_{1}\right)$ to exert neutral prior on $\pi_{i}^{(1)}$. In Pattern 2 ("strong acceptance"), $\kappa_{i}=2$, i.e., both neighboring blocks are nonzero, and the $i$ th block would take a nonzero value with a high probability. Therefore, we use $e_{2}>g_{2}$ in $\operatorname{Beta}\left(e_{2}, g_{2}\right)$ to encourage a high value of $\pi_{i}^{(2)}$ to accept this block.

According to the above generative model, we acquire the analytical posterior distributions of both $\mathbf{z}$ and $\boldsymbol{\eta}^{(l)}$, due to conjugate property. The marginal distribution of $z_{i}$ is expressed as,

$$
p\left(z_{i}=1 \mid-\right)=\left(1+e^{-a_{0}}\right)^{-1}
$$

with

$$
\begin{aligned}
a_{0}= & \frac{1}{2} \sum_{l=1}^{L}\left(\log \beta_{i}-\log \sigma_{i l}+\sigma_{i l} \alpha_{l}^{2} \mathbf{x}_{\backslash i l}^{H} b_{i l} b_{i l}^{H} \mathbf{x}_{\backslash i l}\right) \\
& +\log \pi_{i}-\log \left(1-\pi_{i}\right)
\end{aligned}
$$

where $\sigma_{i l}=\left(\alpha_{l} b_{i l}^{H} b_{i l}+\beta_{i}\right)^{-1}, \mathbf{x}_{\backslash i l}=\mathbf{x}_{l}-\sum_{k \neq i} b_{k l} z_{k} \eta_{k l}$, and $b_{i l}$ is the $i$ th column of the measurement matrix $\mathbf{B}^{(l)}$.

For $z_{i}=1$, the posterior conditional distribution of $p\left(\eta_{i}^{(l)} \mid-\right)$ can be expressed as

$$
p\left(\eta_{i l} \mid-\right)=\mathcal{N}\left(\eta_{i l} \mid \sigma_{i l} \alpha_{l} b_{i l}^{H} \mathbf{x}_{\backslash i l}, \sigma_{i l}\right) .
$$

For $z_{i}=0$, because the value of $\eta_{i l}$ does not affect the result of $r_{i l}$, we conveniently draw the value of variable $\eta_{i l}$ from its prior. Once both $\boldsymbol{\eta}^{(l)}$ and $\mathbf{z}$ are acquired, we can easily obtain the scattering coefficients $\mathbf{r}^{(l)}$ according to Eq. (9).

\section{SIMULATION RESULTS}

For illustrative purposes, two examples are considered in this section. The hyper-parameters are chosen as $\left(e_{0}, g_{0}\right)=$ $(1 /(M N), 1-1 /(M N))$ for strong rejection, $\left(e_{1}, g_{1}\right)=$ $(1 /(M N), 1 /(M N))$ for weak rejection, and $\left(e_{1}, g_{1}\right)=$ $(1-1 /(M N), 1 /(M N))$ for strong acceptance. $Q=2$ nonparametric coherent signals with respective angular spans of $[-32,-26]$ and $[20,25]$ degrees are considered. Only a

Fig. 4. Estimated spatial spectra $(M N=96$, single frequency band).

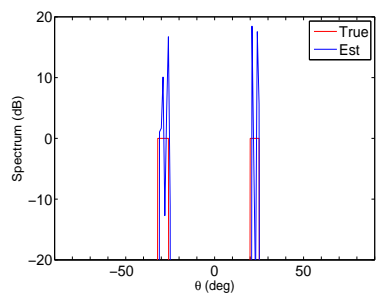

(a) Single frequency band

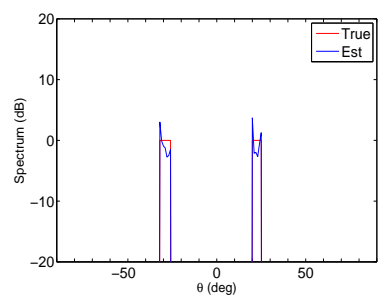

(b) Multiple frequency bands
Fig. 5. Estimated spatial spectra $(M N=32)$.

single snapshot is used in the presence of noise with a $0 \mathrm{~dB}$ signal-to-noise ratio (SNR) for each signal arrival.

In the first example, we compare the performance of the proposed approach with spatial smoothing MUSIC. A MIMO system with $M=8$ transmit and $N=12$ receive antennas is considered for both cases and the results are depicted in Fig. 4. The 20 physical antennas yield a sum coarray with $M N=96$ virtual sensors, and single frequency band waveforms are exploited. It is clear that the proposed approach faithfully estimates the spatial extent and magnitude of both targets, whereas the spatial smoothing MUSIC only identifies disconnected peaks within each angular spreading region, and false peaks are observed.

Next, the performance of proposed methods using single and three mutually coprime frequencies is examined in Fig. 5 with a MIMO system of $M=4$ and $N=8$. Thus, 12 physical antennas yield a 32-element sum coarray. Note that the interelement spacing is differently arranged to the previous example. For illustrative purposes, we choose three mutually coprime frequencies with $L_{1}=1, L_{2}=3$ and $L_{3}=5$. For comparison, the single frequency case only assumes $L_{1}=1$. The estimated spatial spectrum is depicted in Fig. 5(a) from the single frequency case, while the multiple coprime case is illustrated in Fig. 5(b). It is evident that the latter outperforms the former because of the multiple frequency measurements. The multiple coprime frequencies achieve similar performance to Fig. 4(b) with only half of the physical antennas.

\section{CONCLUSIONS}

We proposed an effective approach to estimate the DOA information of multiple nonparametric spatially spreading targets. In a Bayesian compressive sensing framework, the spatial extent of the targets and the multiple frequency band observations are respectively treated as intra- and inter-task dependencies, and effective DOA estimation is achieved through the exploitation of a paired spike-and-slab prior. The superior performance of the proposed algorithm was verified using simulation results. 


\section{REFERENCES}

[1] R. O. Schmidt, "Multiple emitter location and signal parameter estimation," IEEE Trans. Antennas Propagat., vol. 34, no. 3, pp. 276-280, March 1986.

[2] R. Roy and T. Kailath, "ESPRIT - Estimation of signal parameters via rotation invariance techniques," IEEE Trans. Acoust., Speech, Signal Proc., vol. 17, no. 7, pp. 984-995, July 1989.

[3] T.-J. Shan, M. Wax, and T. Kailath, "On spatial smoothing for direction-of-arrival estimation of coherent signals," IEEE Trans. Acoust., Speech Signal Process., vol. 33, no. 4, pp. 806811, Aug. 1985.

[4] J. Li and P. Stoica (Eds.), MIMO Radar Signal Processing. New York, NY: Wiley, 2009.

[5] J. S. Jeong, K. Sakaguchi, J. Takada, and K. Araki, "Performance of MUSIC and ESPRIT for joint estimation of DOA and angular spread in slow fading environment," IEICE Trans. Commun., vol. 85, no. 5, pp. 972-977, May 2002.

[6] S. Valaee, B. Champagne, and P. Kabal, "Parametric localization of distributed source," IEEE Trans. Signal Proc., vol. 43, no. 9, pp. 2144-2153, Sept. 1995.

[7] T. Trump and B. Ottersten, "Estimation of nominal direction of arrival and angular spread using an array of sensors," Signal Proc., vol. 50, no. 1, pp. 57-69, Apr. 1996.

[8] Y. Meng, P. Stoica, and M. Wong, "Estimation of the directions of arrival of spatially dispersed signals in array processing," IEE Proc.-Radar, Sonar and Navig., vol. 143, no. 1, pp. 1-9, Feb. 1996

[9] K. Han and A. Nehorai, "Distributed source processing with linear nested arrays," in Proc. IEEE Sensor Array and Multichannel Signal Proc. Workshop, A Coruña, Spain, June 2014.

[10] Y. U. Lee, J. Choi, I. Song, and S. R. Lee, "Distributed source modeling and direction-of-arrival estimation techniques," IEEE Trans. Signal Proc., vol. 45, no. 4, pp. 960-969, Apr. 1997.

[11] D. Astely and B. Ottersten, "The effects of local scattering on direction of arrival estimation with MUSIC," IEEE Trans. Signal Proc., vol. 47, no. 12, pp. 3220-3234, Dec. 1999.

[12] O. Besson and P. Stoica, "Decoupled estimation of DOA and angular spread for a spatially distributed source," IEEE Trans. Signal Proc., vol. 48, no. 7, pp. 1872-1882, Jul. 2000.

[13] D. L. Donoho, "Compressed sensing," IEEE Trans. Info. Theory, vol. 52, no. 4, pp. 1289-1306, 2006.

[14] Y. D. Zhang, M. G. Amin, F. Ahmad, and B. Himed, "DOA estimation using a sparse uniform linear array with two CW signals of co-prime frequencies," in Proc. IEEE Int. Workshop on Comp. Adv. in Multi-Sensor Adaptive Proc., Saint Martin, Dec. 2013.

[15] S. Qin, Y. D. Zhang, and M. G. Amin, "DOA estimation exploiting coprime frequencies," in Proc. SPIE Wireless Sensing, Localization, and Proc. Conf., Baltimore, MD, May 2014.

[16] A. Bouzerdoum, F. H. C. Tivive, and V. H. Tang, "Multipolarization through-the-wall radar imaging using joint Bayesian compressed sensing," in Proc. Int. Conf. Digital Sig. Proc., Hong Kong, China, Aug. 2014.

[17] Q. Wu, Y. D. Zhang, M. G. Amin, and B. Himed, "Multi-static passive SAR imaging based on Bayesian compressive sensing," in Proc. SPIE Compressive Sensing Conf., Baltimore, MD, May 2014.

[18] G. Li, P. K. Varshney, and Y. D. Zhang, "Multistatic radar imaging via decentralized and collaborative subspace pursuit," in Proc. Int. Conf. Digital Sig. Proc., Hong Kong, China, Aug. 2014.
[19] Q. Wu, Y. D. Zhang, M. G. Amin, and F. Ahmad, "Throughthe-wall radar imaging based on modified Bayesian compressive sensing," in Proc. IEEE China Summit and Int. Conf. Signal and Inform. Proc., Xi' an, China, July 2014.

[20] Q. Wu, Y. D. Zhang, M. G. Amin, and F. Ahmad, "Robust multipath exploitation radar imaging in urban sensing based on Bayesian compressive sensing," in Proc. Asilomar Conf. Signals, Systems, and Computers, Pacific Grove, CA, Nov. 2014.

[21] Q. Wu, Y. D. Zhang, M. G. Amin, and B. Himed, "Multitask Bayesian compressive sensing exploiting intra-task correlation," IEEE Signal Proc. Lett., vol. 22, no. 4, pp. 430-434, April 2015.

[22] S. Ji, D. Dunson, and L. Carin, "Multitask compressive sensing," IEEE Trans. Signal Proc., vol. 57, no. 1, pp. 92-106, 2009.

[23] Z. Zhang and B. D. Rao, "Extension of SBL algorithms for the recovery of block sparse signals with intra-block correlation," IEEE Trans. Signal Proc., vol. 61, no. 8, pp. 2009-2015, Apr. 2013.

[24] Q. Wu, Y. D. Zhang, M. G. Amin, and B. Himed, "Complex multitask Bayesian compressive sensing," in Proc. IEEE ICASSP, Florence, Italy, May 2014.

[25] M. Carlin, P. Rocca, G. Oliveri, F. Viani, and A. Massa, "Directions-of-arrival estimation through Bayesian compressive sensing strategies," IEEE Trans. Antennas Propagat., vol. 61, no. 7, pp. 3828-3838, July 2013.

[26] M. G. Amin, Y. D. Zhang, and B. Jokanovic, "Time-frequency signature reconstruction from random observations using multiple measurement vectors," in Proc. IEEE ICASSP, Florence, Italy, May 2014.

[27] Q. Wu, Y. D. Zhang, and M. G. Amin, "Continuous structure based Bayesian compressive sensing for sparse reconstruction of time-frequency distributions," in Proc. Int. Conf. Digital Sig. Proc., Hong Kong, China, Aug. 2014.

[28] Y. D. Zhang and B. Himed, "Space-time adaptive processing in bistatic passive radar exploiting complex Bayesian learning," in Proc. IEEE Radar Conf., Cincinnati, OH, May 2014.

[29] Q. Wu, Y. D. Zhang, M. G. Amin, and B. Himed, "Spacetime adaptive processing in multi-static passive radar exploiting group sparsity," in Proc. IEEE Int. Radar Conf., Arlington, VA, May 2015.

[30] R. T. Hoctor and S. A. Kassam, "The unifying role of the coarray in aperture synthesis for coherent and incoherent imaging," Proc. IEEE, vol. 78, no. 4, pp. 735-752, April 1990.

[31] P. Pal and P. P. Vaidyanathan, "Nested arrays: A novel approach to array processing with enhanced degrees of freedom," IEEE Trans. Signal Proc., vol. 58, no. 8, pp. 4167-4181, Aug. 2010.

[32] S. Qin, Y. D. Zhang, and M. G. Amin, "DOA estimation of mixed coherent and uncorrelated signals exploiting a nested MIMO system," in Proc. IEEE Benjamin Franklin Symp. Microwave and Antenna Sub-systems, Philadelphia, PA, Sept. 2014.

[33] S. Qin, Y. D. Zhang, and M. G. Amin, "Generalized coprime array configurations," in Proc. IEEE Sensor Array and Multichannel Signal Proc. Workshop, A Coruña, Spain, June 2014.

[34] S. Qin, Y. D. Zhang, and M. G. Amin, "Generalized coprime array configurations for direction-of-arrival estimation," IEEE Trans. Signal Proc., in press.

[35] L. Yu, J. P. Barbot, G. Zheng, and H. Sun, "Compressive sensing for cluster structured sparse signals: Variational Bayes approach," Technical Report, 2011. Available at http://hal. archives-ouvertes.fr/docs/00/57/39/53/PDF/cluss_vb.pdf. 